\title{
MCRS1 is essential for epiblast development during early mouse embryogenesis
}

\author{
Wei Cui ${ }^{1,2}$, Agnes Cheong1, Yongsheng Wang³, Yuran Tsuchida1, Yong Liu ${ }^{4}$ \\ Kimberly D Tremblay ${ }^{1}$ and Jesse Mager ${ }^{1}$ \\ ${ }^{1}$ Department of Veterinary and Animal Sciences, University of Massachusetts, Amherst, Massachusetts, USA, \\ ${ }^{2}$ Animal Models Core Facility, Institute for Applied Life Sciences (IALS), University of Massachusetts, Amherst, \\ Massachusetts, USA, ${ }^{3}$ Key Laboratory of Animal Biotechnology of the Ministry of Agriculture, College of Veterinary \\ Medicine, Northwest A\&F University, Yangling, Shaanxi, People's Republic of China and ${ }^{4}$ Anhui Province Key \\ Laboratory of Embryo Development and Reproductive Regulation, Anhui Province Key Laboratory of Environmental \\ Hormone and Reproduction, Fuyang Normal University, Fuyang, Anhui, People's Republic of China
}

Correspondence should be addressed to W Cui or J Mager; Email: wcui@umass.edu or jmager@vasci.umass.edu

\begin{abstract}
Microspherule protein 1 (MCRS1, also known as MSP58) is an evolutionarily conserved protein that has been implicated in various biological processes. Although a variety of functions have been attributed to MCRS1 in vitro, mammalian MCRS1 has not been studied in vivo. Here we report that MCRS1 is essential during early murine development. Mcrs1 mutant embryos exhibit normal morphology at the blastocyst stage but cannot be recovered at gastrulation, suggesting an implantation failure. Outgrowth (OG) assays reveal that mutant blastocysts do not form a typical inner cell mass (ICM) colony, the source of embryonic stem cells (ESCs). Surprisingly, cell death and histone $\mathrm{H} 4$ acetylation analysis reveal that apoptosis and global $\mathrm{H} 4$ acetylation are normal in mutant blastocysts. However, analysis of lineage specification reveals that while the trophoblast and primitive endoderm are properly specified, the epiblast lineage is compromised and exhibits a severe reduction in cell number. In summary, our study demonstrates the indispensable role of MCRS1 in epiblast development during early mammalian embryogenesis.

Reproduction (2020) 159 1-13
\end{abstract}

\section{Introduction}

Mammalian preimplantation embryo development begins with oocyte fertilization and concludes with the formation of a blastocyst-stage embryo that is capable of uterine implantation (Arny et al. 1987). During this period, the newly formed embryo undergoes three major transcriptional and morphogenic events. The first event, maternal-to-zygotic transition (MZT), initiates the epigenetic reprogramming that is required to establish totipotency (Latham et al. 1991, Liu et al. 2018). Following several early cleavage divisions, the eight-cell embryo initiates compaction and polarization, events critical for the first cell-fate acquisition (Sutherland \& Calarco-Gillam 1983, Houliston \& Maro 1989). The last major preimplantation event occurs when the polarized outer cells of compacted morula differentiate into the trophectoderm (TE) of the blastocyst, while the apolar cells located inside develop into the inner cell mass (ICM) (Nichols et al. 1998, Tompers et al. 2005). Welldefined gene expression profiles occur within these two distinct lineages to maintain and reinforce cell fate. For example, the transcription factor (TF) CDX2 is enriched in TE, whereas the TF OCT4 (alias POU5F1) becomes highly expressed in the ICM (Niwa et al. 2005). At approximately the 32 cell stage, the ICM becomes segregated into epiblast (EPI) and primitive endoderm $(\mathrm{PE})$, demarcated by NANOG expression in the EPI cells and SOX17 in the PE (Strumpf et al. 2005). These first three lineages $E P I, P E$, and TE will give rise to the embryo, parietal yolk sac, and placenta, respectively.

Lineage specification during early embryogenesis is a highly regulated process that relies on the differential expression of various genes within distinct cell populations. In addition to key TFs, such as NANOG and CDX2, many additional genes required for specific lineage function have been identified through largescale RNAi screening and genome editing (Cao et al. 2015, Cui et al. 2016, 2018). By using an advanced single-cell gene-expression and 3D mapping analysis, microspherule protein 1 (MCRS1) was recently identified as a putative EPI-specific marker in human blastocysts (Durruthy-Durruthy et al. 2016). Remarkably, overexpression of MCRS1 as well as two other novel EPI markers TET1 and THAP11, leads to the resetting of primed human embryonic stem cells (hESCs) to naive pluripotency in vitro (Durruthy-Durruthy et al. 2016). 
The role of MCRS1 has not yet been assessed during human development due to limitations of working with human embryos.

MCRS1 (also called MSP58 or P78) is an evolutionarily conserved protein that was initially identified in yeast as a nucleolar protein associated with rRNA transcription and cell proliferation (Ren et al. 1998). Later in both yeast and humans, MCRS1 was found to act as the regulatory component of the chromatin remodeling INO80 complex, catalyzing ATP-dependent sliding of nucleosomes along DNA (Jin et al. 2005). In both Drosophila and human, MCRS1 is a subunit of the non-specific lethal (NSL) Complex, which can acetylate nucleosomal histone $\mathrm{H} 4$ on multiple lysine residues, including lysine 5 (H4K5), 8 (H4K8), and 16 (H4K16) (Cai et al. 2010). Interestingly, in Drosophila, MCRS1 promotes gene transcription by facilitating the recruitment of the RNA polymerase II (RNAP II) preinitiation complexes to the promoter regions of target genes (Andersen et al. 2010). In human cell lines, MCRS1 has also been implicated in proliferation, inducing senescence, mitotic spindle assembly, activation of mechanistic target of rapamycin complex 1 (mTORC1), and tumorigenesis (Benavides et al. 2013, Liu et al. 2014, Fawal et al. 2015). However, the function of MCRS1 during mammalian development has not been studied.

In the present study, we use both knockout (KO) and knockdown (KD) strategies to explore the role of MCRS1 during murine development. Our data show that MCRS1 is essential for early embryo survival and successful implantation due to a specific role in epiblast function and development during the initial stages of lineage commitment and expansion.

\section{Materials and methods}

Unless otherwise specified, all chemicals and media were obtained from Millipore-Sigma.

\section{Generation of Mcrs1 mutants}

All animal experimental protocols were approved by the Institutional Animal Care and Use Committee of the University of Massachusetts, Amherst (2015-0011, 2018-0003). Mcrs 1

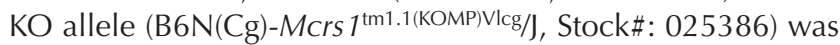
generated on $\mathrm{C} 57 \mathrm{BL} / 6 \mathrm{NJ}$ background by the Knockout Mouse Phenotyping Program (KOMP2) at The Jackson Laboratory (JAX). Briefly, a beta-galactosidase containing ZEN-UB1 Velocigene cassette (Cassette\#: VG16025) was inserted into the Mcrs 1 gene, replacing all coding exons and intervening sequences (Fig. 1A) and creating a deletion of 7372 bp between positions 99,250,469 - 99,243,098 of Chromosome 15 (Genome Build 38). The construct was introduced into C57BL/6N-derived VGB6 embryonic stem cells and the resulting chimeric males bred to $\mathrm{C} 57 \mathrm{BL} / 6 \mathrm{NJ}$ females. Offspring were crossed to B6N.Cg-Tg(Sox2-cre) $1 \mathrm{Amc} / \mathrm{J}$ to remove the neo cassette and the offspring were crossed to remove the transgene. To expand the line in our colony, heterozygous (Het) mice from JAX were backcrossed again with C57BL/6NJ WT. Heterozygous intercrosses were used to generate Mcrs 1 mutants (Mut). The genotyping primers include WT Forward: CCCTGACTTGGGTCAGAGTT; WT Reverse: CTGCATCTGAGAAGTTGAGCA; Mut Forward: CGGTCGCTACCATTACCAGT; Mut Reverse: CTTGCCTCAAA GGCTCAATC.

\section{Embryo recovery, culture and genotyping}

For natural matings, Mcrs 1 heterozygous females 8 to 14 weeks old were caged with Mcrs 1 heterozygous males and the presence of a vaginal plug defined as embryonic day 0.5 (E0.5). Embryos were collected from heterozygous females by dissection or flushing to collect E7.5 or E3.5 embryos, respectively. Embryos were imaged, collected into individual tubes and then lysed for PCR genotyping.

To prepare zygotes for siRNA microinjection or in vitro culture and immunofluorescence, B6D2F1 female mice 8 to 10 weeks old were super-ovulated with $7.5 \mathrm{IU}$ pregnant mare serum gonadotropin (PMSG, BioVendor, Asheville, NC, USA), followed $48 \mathrm{~h}$ later by $7.5 \mathrm{IU}$ human chorionic gonadotropin (hCG). Females were then mated with B6D2F1 males and euthanized $20 \mathrm{~h}$ post hCG injection. Zygotes were released from oviducts and cumulus cells were removed by pipetting in M2 medium containing hyaluronidase. Zygotes were washed in $\mathrm{M} 2$ and cultured in $\mathrm{KSOM}$ at $37^{\circ} \mathrm{C}$ in a humidified atmosphere of $5 \% \mathrm{CO}_{2}, 5 \% \mathrm{O}_{2}$ balanced in $\mathrm{N}_{2}$.

\section{Outgrowth assay}

Blastocysts were harvested and cultured individually in a single well of DMEM (Lonza, Allendale, NJ, USA) containing $10 \%$ fetal bovine serum (Atlanta Biologicals, Flowery Branch, GA, USA) and $1 X$ GlutaMAX (Thermo Fisher Scientific). Blastocysts were allowed to attach and outgrow for 3 days at $37^{\circ} \mathrm{C}$ in a humidified atmosphere of $5 \% \mathrm{CO}_{2}$ prior to imaging and genotyping. Outgrowths were evaluated by morphology as previously demonstrated (Cui et al. 2016, 2018). Briefly, an outgrowth that displayed a distinctive ICM colony surrounded by trophoblast monolayer is considered a normal successful outgrowth, while outgrowths that fail to hatch, lack ICM colony or lack trophoblast monolayer are considered as failed outgrowths.

\section{Immunofluorescence and imaging of preimplantation embryos}

Immunofluorescence (IF) was performed as previously described (Cui et al. 2018). E3.5 blastocysts were freshly harvested by flushing and then cultured overnight at $37^{\circ} \mathrm{C}$ in a humidified atmosphere of $5 \% \mathrm{CO}_{2}, 5 \% \mathrm{O}_{2}$ balanced in $\mathrm{N}_{2}$ prior to fixation and IF (to ensure embryos had undergone EPI/PE/ TE specification). In vitro-produced blastocysts were harvested at 4 days post microinjection. All primary antibodies for IF were used at 1:200 including mouse anti-CDX2 (BioGenex, MU392A-UC); rabbit anti-NANOG (Abcam, ab80892); rabbit anti-TRP53 (Cell Signaling Technology, \#9284); rabbit 

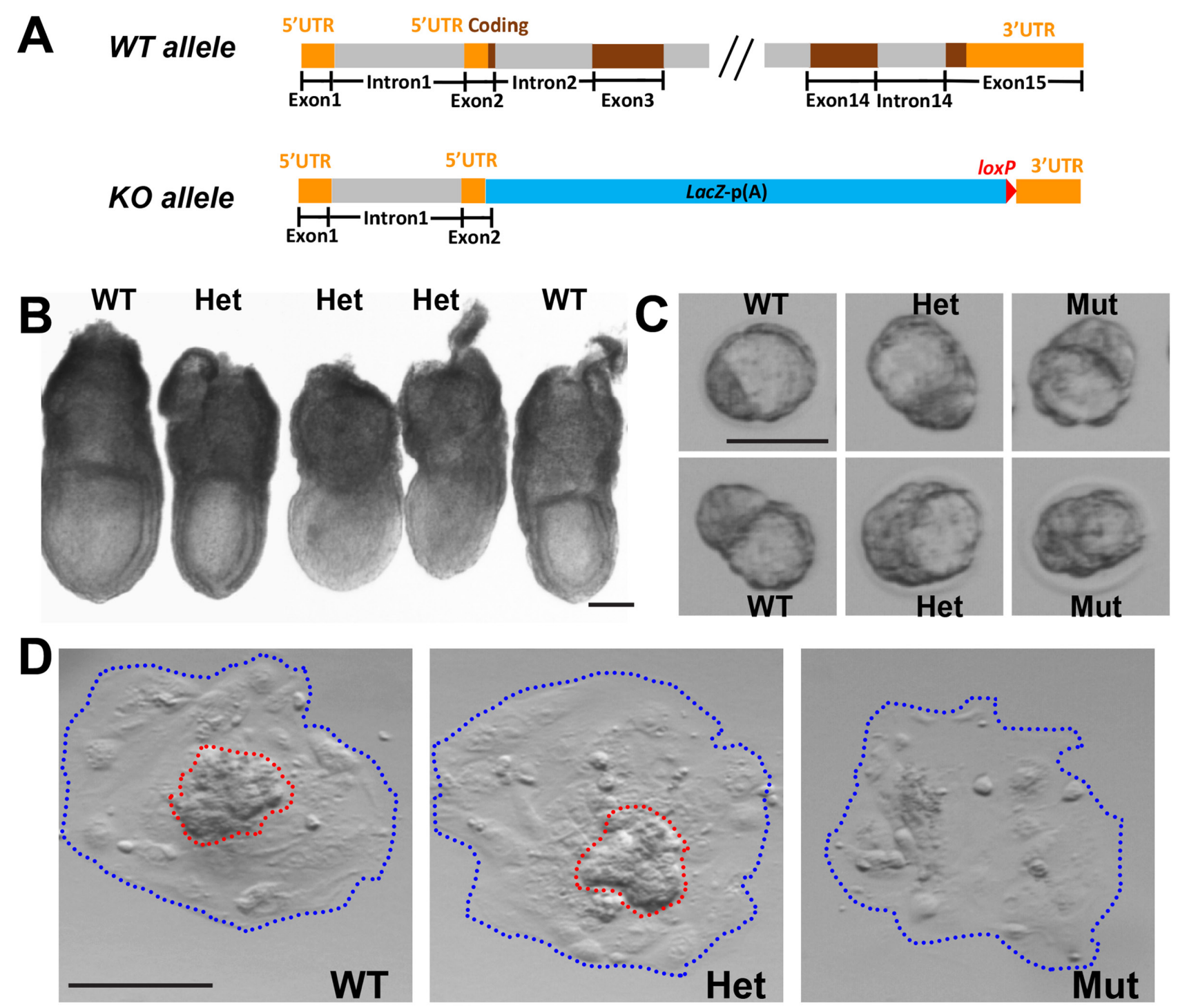

Figure 1 (A) Schematic of Mcrs 1 knockout allele generation. lacZ, $\beta$-galactosidase coding sequence from the $E$. coli lac $Z$ gene; $p(A)$, polyadenylation signal. (B) Representative genotyped embryos at E7.5. (C) Representative genotyped embryos at E3.5. (D) Outgrowths from WT and Het displayed a distinctive ICM (red dashed line) surrounded by trophoblast (blue dashed line), while Mut outgrowths did not display an ICM. Scale bars, $100 \mu \mathrm{m}$.

anti-MCRS1 (Sigma-Aldrich, HPA039057); rabbit antiH4K5 + K8 + K12 + K16 (Abcam, ab177790); goat anti-SOX17 (R\&D Systems, AF1924); goat anti-OCT4 (Abcam, ab27985). After suitable secondary antibody (Alexa Fluor, Thermo Fisher Scientific) and DAPI staining, embryos were individually transferred into single wells of a chambered slide (Corning Co., Corning) for imaging using a Nikon A1 Spectral Detector Confocal with FLIM Module. Z-stacks (20x objective, $8 \mu \mathrm{m}$ sections) were collected and maximum projection applied. Embryos were handled individually such that each one was imaged and then recovered for PCR genotyping (example of individual blastocysts post imaging and genotyping shown in Supplementary Fig. 1, see section on supplementary materials given at the end of this article). Fluorescence intensity of $\mathrm{H} 4$ acetylation was quantified and analyzed as previously described (An et al. 2019). Briefly, high-resolution z-stack images were acquired under identical capture settings, and relative intensities were measured on the raw images using ImageJ software (Schneider et al. 2012), with DAPI or OCT4 intensity as the reference.

\section{X-gal (5-bromo-4-chloro-3-indolyl- $\beta$-D- galactopyranoside) staining}

Freshly dissected embryos were fixed in X-gal buffer containing $0.2 \%$ glutaraldehyde and $1 \%$ formaldehyde on ice for $15 \mathrm{~min}$ and subjected to modifications from previous protocol (Tremblay et al. 2000). In brief, the fixed embryos were washed with X-gal buffer (PBS, $5 \mathrm{mM}$ EGTA, $2 \mathrm{mM}$ MgCl: $6 \mathrm{H}_{2} \mathrm{O}, 0.2 \% \mathrm{NP}-40,0.2 \mathrm{mM}$ deoxycholate) for $10 \mathrm{~min}$ three times and stained with $\mathrm{X}$-gal stain (X-gal buffer with $5 \mathrm{mM}$ potassium ferricyanide, $5 \mathrm{mM}$ potassium ferrocyanide, 
and $0.5 \mathrm{mg} / \mathrm{ml} \mathrm{X}$-gal) for $48 \mathrm{~h}$ at $37^{\circ} \mathrm{C}$. Subsequently, embryos were dehydrated in ethanol, cleared in xylene, embedded in paraffin, and sectioned at $7 \mu \mathrm{m}$. Slides were deparaffinated in xylene, counterstained with Eosin and coverslipped in Cytoseal (Thermo Fisher Scientific) and imaged with a Pannoramic MIDI II slide scanner (3DHISTECH Ltd., Budapest, Hungary).

\section{Microinjection}

Microinjection was performed as previously described (Cui et al. 2016). A volume of 5-10 pL of $50 \mu \mathrm{M}$ Scrambled Control (5'-CAGGGTATCGACGATTACAAA, Qiagen) or Mcrs 1 siRNA (siRNA1 target: 5'-CAAGGTGTCATCAAGTTGAAA, siRNA2 target: 5'-CTCAAGGACATGCGAGATGAA, Qiagen) was microinjected into the cytoplasm of zygotes.

\section{RNA extraction and reverse transcription PCR (RT-PCR)}

Total RNA extraction was performed with a Roche High Pure RNA Isolation Kit (\#11828665001). cDNA was synthesized using iScript cDNA synthesis kit (\#170-8891; Bio-Rad Laboratories). Intron-spanning primers used for RT-PCR include (Actb: 5'-GGCCCAGAGCAAGAGAGGTATCC and 5'-ACGCACGATTTCCСTCTCAGC; Mcrs 1: 5'-GAGCCCTCC TCTAGTGAGAAG and 5'-ACCCAGGTGGACAGATGTCA).

\section{Statistical analysis}

All experiments were repeated at least three times. Percentage data were analyzed by ANOVA, and a value of $P<0.05$ was considered statistically significant. Data are expressed as mean \pm S.E.M.

\section{Results}

\section{Mcrs1 mutants cannot be recovered in vivo after E3.5}

The Mcrs 1 knockout allele was generated by insertion of ZEN-UB1 Velocigene cassette (Cassette\#: VG16025, http://velocigene.com/komp/detail/16025) for the Knockout Mouse Phenotyping Program (KOMP2) at The Jackson Laboratory (Fig. 1A). During the initial phenotyping performed for the International Mouse Phenotyping Consortium (IMPC), no homozygous Mcrs 1 mutants were born, nor found at E15.5 or E12.5 (http:// www.mousephenotype.org). Therefore, we first dissected embryos at E7.5. Forty embryos were recovered from seven heterozygous intercrosses. Genotyping revealed 25 Het and 15 WT embryos (Fig. 1B). No Mcrs 1 homozygous mutant embryos were present at E7.5, nor were there increased numbers of empty decidua $(n=4)$, suggesting that Mcrs 1 mutants fail to implant. We next collected three litters of embryos at E3.5, and recovered four Mcrs 1 mutants, ten Het and two WT embryos at the blastocyst stage. Mutant blastocysts were indistinguishable from littermates based on morphology alone (Fig. 1C).

Since Mcrs 1 mutant blastocysts appear morphologically normal but are not found at E7.5, we performed in vitro outgrowth assays as a surrogate for implantation in vivo which allows for assessment of hatching from the zona pellucida as well as ICM and TE cell growth. Blastocysts were obtained from three heterozygous intercrosses and individual embryos were cultured for 3 days on tissue culture plastic. As expected, hatching and successful outgrowth rates were high for WT $(100 \%, 3 / 3)$ and Het blastocysts (80\%, 8/10). By 72 $\mathrm{h}$ in culture, successful outgrowths display a distinct $3 \mathrm{D}$ ICM colony surrounded by trophoblast cells which are easily identified by their flattened morphology (Fig. 1D). Although each mutant blastocyst hatched and produced trophoblast and primitive endoderm cells, none of the four mutants assayed formed a typical ICM colony. These results suggest that the implantation failure of Mcrs 1 mutant blastocysts is due to impaired signaling from or proliferation of the ICM.

\section{Expression of MCRS1 during early mouse embryogenesis}

Based on the phenotype and timing of lethality, we evaluated MCRS1 expression during preimplantation and gastrulation stages. Immunofluorescence (IF) and RT-PCR analysis of WT preimplantation embryos revealed that Mcrs 1 mRNA and protein are present at all stages examined (Fig. 2A and B). MCRS1 protein is present in both the cytoplasm and nucleus from the oocyte through the two-cell stage. By the four-cell stage, MCRS1 appears more concentrated in the nucleus and by the blastocyst stage MCRS1 becomes notably concentrated in the nuclei of ICM cells compared with the TE (Fig. 2A). In order to validate the antibody, we performed IF on late blastocysts (dissected at E3.5 and cultured overnight to further deplete the maternally loaded protein/mRNA) from Het intercrosses. Each embryo was genotyped after imaging to retrospectively examine the images knowing specific genotypes. MCRS1 protein was observed in both ICM and TE from all WT $(n=6)$ and Het $(n=10)$ blastocysts (possible enriched in the ICM). However, no signal was detected in ICM nor TE of any mutant embryos ( $n=5$, Fig. $2 \mathrm{C}$ ). All embryos regardless of genotype showed non-specific signal on the zona pellucida. Based on the absence of antibody signal specifically in mutant embryos, these results confirm that the knockout allele does not produce MCRS1 protein.

We also examined MCRS1 expression during gastrulation by examining expression of the $L a c Z$ reporter, inserted during generation of the knockout allele, in Hets. At E6.5, LacZ is weak but present throughout cells of the epiblast (Epi) and extra-embryonic ectoderm (Exe) cells (Fig. 2D and $\mathrm{D}^{\prime}$ ) but is largely absent from the visceral endoderm (VE). At E7.5, this same lineagespecific pattern continues (Fig. 2D and D"). Together, the LacZ-staining pattern observed during gastrulation is consistent with the ICM enriched expression observed in blastocysts (Fig. 2A). 

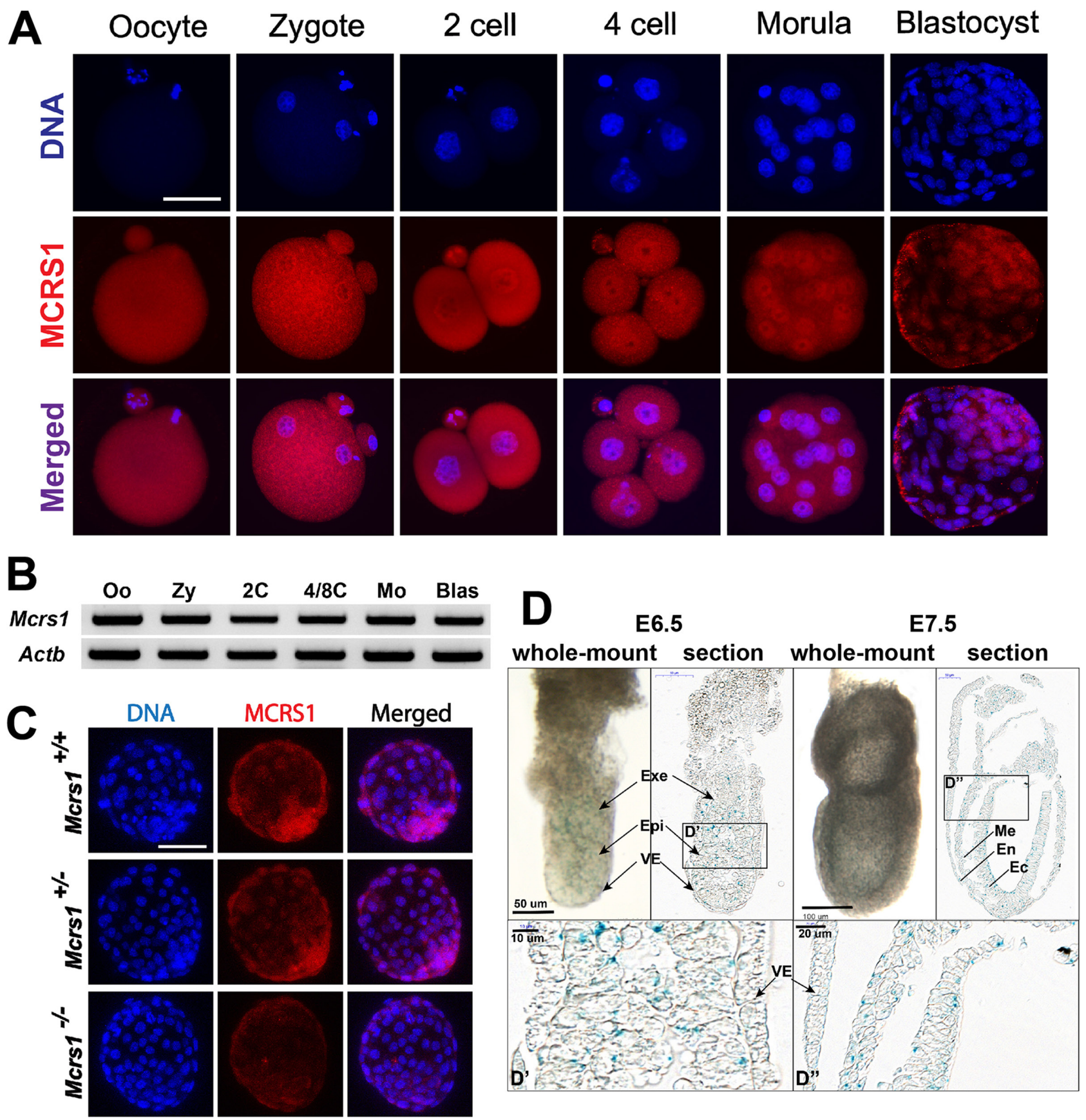

Figure 2 (A) Immunofluorescence (IF) identifying MCRS1 protein expression from the metaphase II oocyte to the blastocyst stage. Scale bar, 50 $\mu \mathrm{m}$. (B) RT-PCR to identify Mcrs 1 expression in WT pre-implantation embryos. Actb was used as loading control. Oo, metaphase II oocyte; Zy, zygote; 2C, 2-cell embryo; 4/8C, mix of 4- and 8-cell stage embryos; Mo, morula; Blas, Blastocyst. (C) WT and Het blastocysts express MCRS1 protein in both ICM and TE, while no signal is detected in homozygous mutants (all embryos show non-specific zona staining). Scale bar, 50 $\mu \mathrm{m}$. (D) LacZ-reporter staining of both E6.5 and E7.5 Hets. Exe, extra-embryonic ectoderm; Epi, epiblast; VE, visceral endoderm. Ec, ectoderm; Me, mesoderm; En, endoderm. D' and D": enlarged LacZ staining showing that LacZ is present throughout cells of the Epi and Exe cells but is largely absent from the VE.

\section{Mcrs1 mutants have fewer ICM cells}

To explore the mechanism of embryonic lethality in vivo and outgrowth failure in vitro, we first examined markers of apoptosis (active TRP53) and the first cell lineage specification (OCT4 for ICM and CDX2 for TE). Of 20 blastocysts examined from heterozygous intercrosses (4 WT, 12 Het, 4 Mut), none showed appreciable levels of active TRP53, indicating no increase in cell death in Mcrs 1 mutant blastocysts (Fig. $3 \mathrm{~A}$ and Supplementary Fig. 2). All embryos exhibited OCT4 expression specifically 
in the ICM and mutually exclusive CDX2 expression in the TE. Although OCT4 expression was confined to a cluster of cells within Mcrs $1^{-1-}$ blastocysts, there is an obvious reduction in the number of OCT4-positive cells compared with Het and WT littermates (Fig. 3A). Further quantification of several litters of embryos demonstrated that although blastocysts of all genotypes (9 WT, 26 Het, 10 Mut) have a similar total cell number (Fig. 3B), the percentage of OCT4-positive cells is severely reduced in mutant blastocysts (Fig. 3C). Concordant with reduced
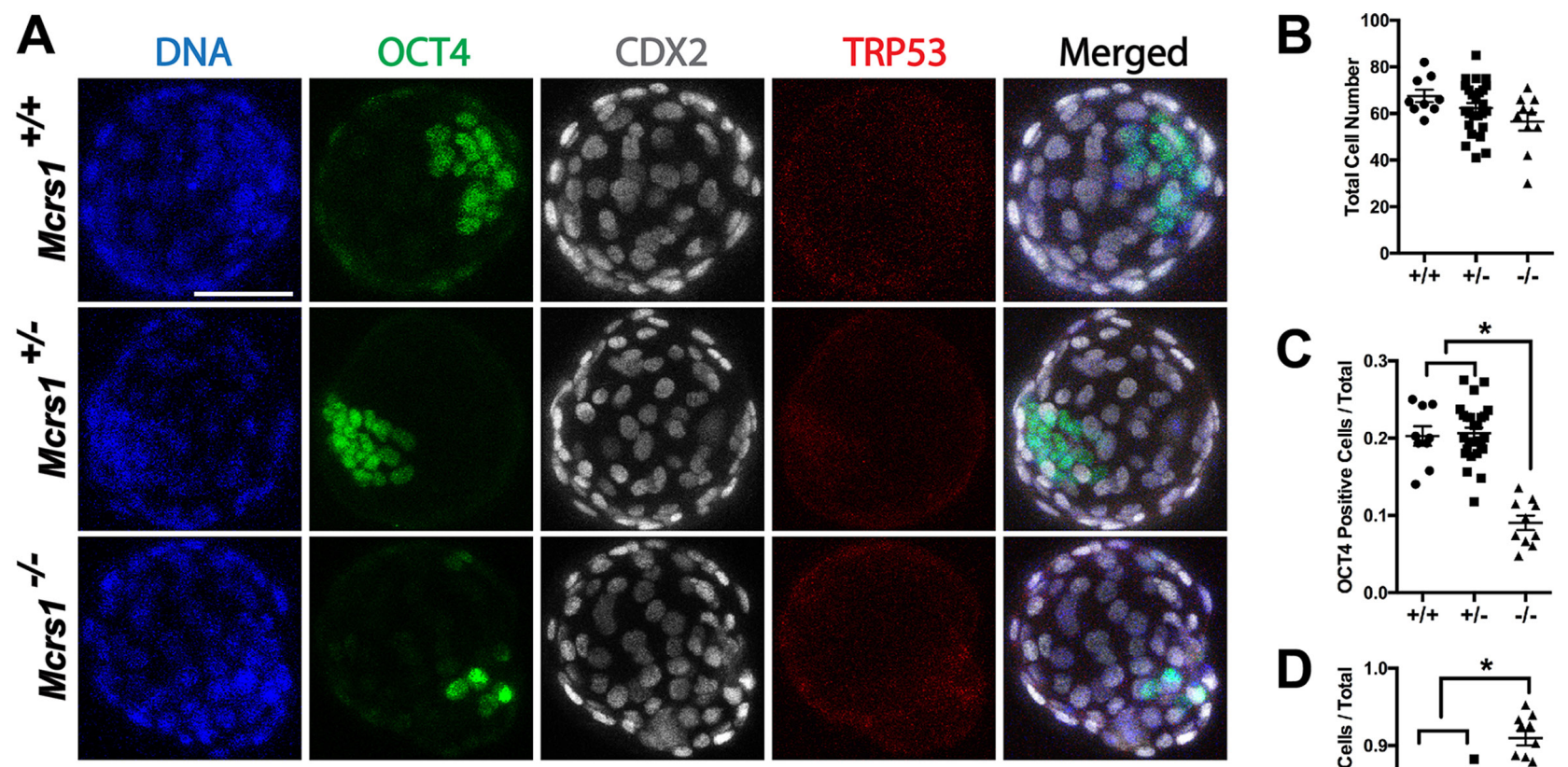

C
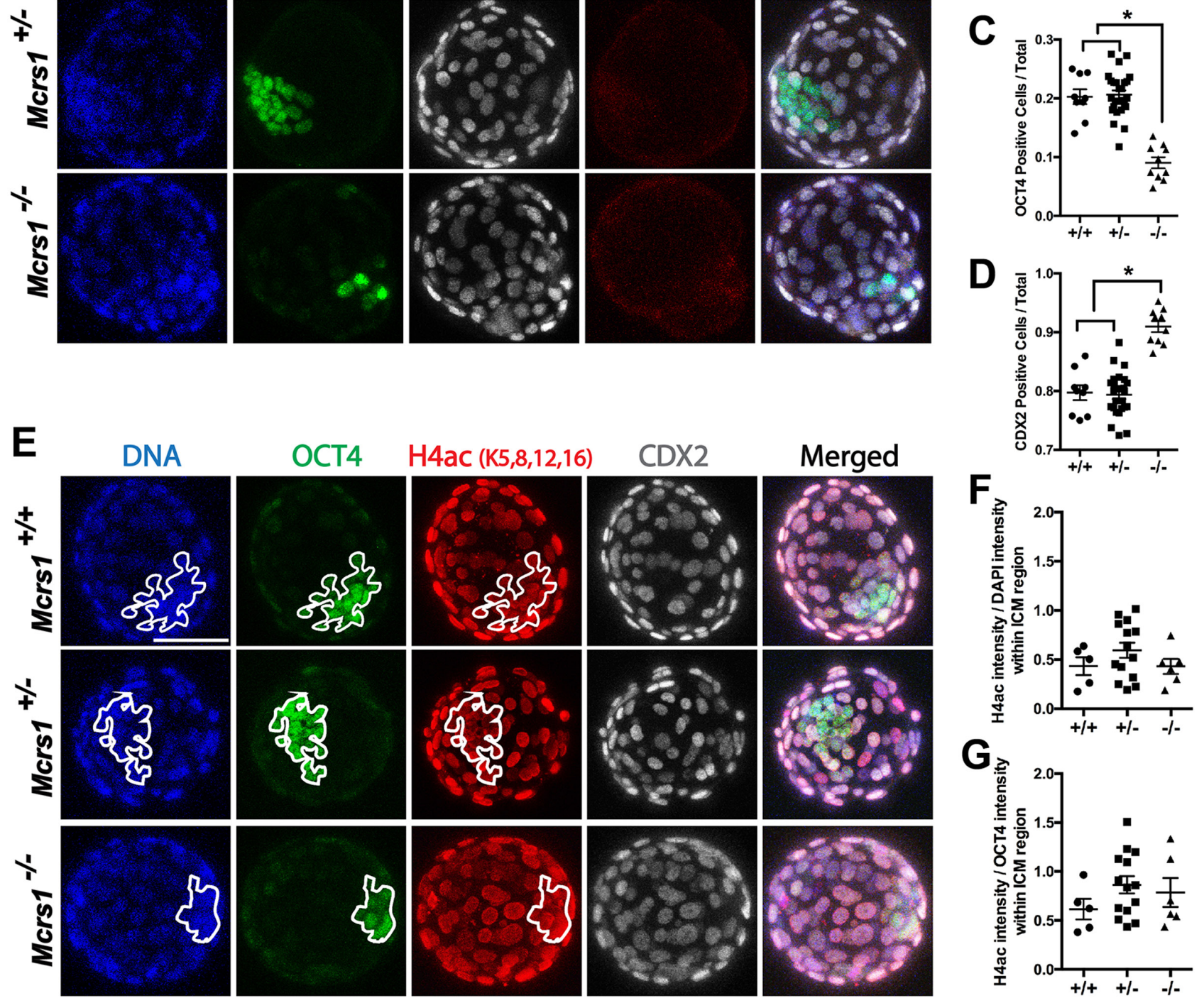

Figure 3 (A) IF of OCT4 (ICM marker), CDX2 (TE marker), and active TRP53 (apoptosis marker) in blastocysts of different genotypes. (B) All genotypes had similar total cell number per blastocyst. (C) The percentage of ICM cells (OCT4 positive) in Mut embryos was significantly decreased and (D) the percentage of CDX2-positive TE cells in Mut embryos was significantly increased when compared with WT and Het littermates. Embryos evaluated in Fig. 3B, C and D: 9 WT, 26 Het, 10 Mut. (E) H4 acetylation (antibody recognizing H4K5+K8+K12+K16) in blastocysts of different genotypes. Fluorescence intensity analysis of $\mathrm{H} 4$ acetylation in ICM cells (white outlines) showed no significant difference between genotypes when DAPI intensity (F) or OCT4 (G) was used as reference. Embryos evaluated in Fig. 3F and G: 5 WT, 14 Het, 6 Mut. Scale bars, $50 \mu \mathrm{m} .{ }^{*} P<0.05$. 
OCT4 positive cell number, an increase in the percent of CDX2-positive cells is also evident (Fig. 3D). These results indicate that ICM and TE are both specified in the absence of Mcrs 1, but that the ratio of cell allocation to each lineage is altered with only $45 \%$ of the appropriate number of ICM cells present in mutants.

Considering this and the possible role of MCRS1 in histone $\mathrm{H} 4$ acetylation on multiple lysine residues (Cai et al. 2010), another batch of blastocysts (5 WT, 14 Het, 6 Mut) was evaluated for $\mathrm{H} 4$ acetylation levels, using an antibody that recognizes multiple $\mathrm{H} 4 \mathrm{ac}$ residues $(\mathrm{H} 4 \mathrm{~K} 5+\mathrm{K} 8+\mathrm{K} 12+\mathrm{K} 16)$. As shown in Fig. 3E and $\mathrm{F}$, no significant difference was observed in the H4ac levels in ICM cells among different genotypes indicating there is no global loss of H4ac in MCRS1 mutants. Since we observed a reduction in ICM cell number (Fig. $3 \mathrm{~A}$ and C), we also calculated $\mathrm{H} 4 \mathrm{ac}$ intensity/OCT4 intensity to see if there is any correlation between $\mathrm{H} 4 \mathrm{ac}$ and OCT4 expression in the absence of MCRS1. As shown in Fig. 3G, no significant difference was identified among different genotypes.

\section{Mcrs1 mutants have normal PE but defective EPI allocation}

To further explore the phenotype in Mcrs 1 mutant blastocysts, we investigated the fidelity of the second embryonic lineage decision, ICM segregation into epiblast (EPI) and primitive endoderm (PE). Blastocysts flushed from heterozygous intercrosses were assessed for the EPI marker NANOG and the PE marker SOX17 (Fig. 4A). From all 26 blastocysts genotyped (4 WT, 15 Het, 7 Mut), we found that Mut blastocysts contained a significantly reduced number and percentage of EPI cells (NANOG positive) when compared with WT and Het embryos (Fig. 4B and C). However, both the total number of PE cells per blastocyst (SOX17 positive, Fig. 4D) and the percentage of PE cells (Fig. 4E) were not significantly altered in Mcrs 1 mutants. Of the seven Mut blastocysts collected for this experiment, four had very few EPI cells, while other three (Fig. 4A, bottom row) contained no NANOG-positive cells within the ICM.

\section{Mcrs1 knockdown embryos phenocopy genetic-knockout mutants}

In order to establish a more efficient platform for studying the function of MCRS1 during development, we used RNA interference (RNAi) to knockdown (KD) Mcrs 1. Two distinct Mcrs 1 siRNAs were individually microinjected into zygotes. Robust KD efficacy was confirmed at the early morula stage after microinjection with both siRNAs as evidenced by a loss of MCRS1 protein IF (Fig. 5A) and mRNA (Fig. 5B). Similar to the KO phenotype, Mcrs 1 KD did not affect blastocyst formation or morphology (Fig. 5C and G) but did lead to outgrowth failure, where all failed KD outgrowths exhibited complete lack of morphologically evident ICM colony (Fig. 5G). Importantly, similar to the KO, KD with either siRNA did not change ratio of SOX17-positive PE cells (Fig. 5D), but did lead to a decrease in the ratio of NANOG-positive EPI cells (Fig. 5E), and concordantly an increase in the ratio of CDX2-positive TE cells (Fig. 5F), compared with control blastocysts (evaluated embryos: control $=13$, siRNA1 $=13$, siRNA2 =12). We speculated that a more severe KD phenotype might be achieved with extended culture time. As expected, when KD and control embryos were subjected to an additional 72-h outgrowth, a more severe phenotype was observed. Embryos that did not hatch during in vitro outgrowth had zero NANOG-positive cells in KD groups $(0 / 21$, siRNA1 =10, siRNA2 =11), while all embryos from the control group displayed robust NANOG-positive cells (11/11) (Fig. 6). For those KD embryos that did hatch during in vitro outgrowth, again, no NANOGpositive cells could be detected in KD outgrowths $(0 / 20$, $\operatorname{siRNA} 1=10$, siRNA2 $=10)$, whereas majority of the control outgrowths exhibited strong NANOGpositive cells (12/15) (Fig. 6). Furthermore, no obvious differences were noted in SOX17 or CDX2 expression in KD outgrowths (Fig. 6, additional examples shown in Supplementary Fig. 3). These results show that the phenotypes of Mcrs $1 \mathrm{KO}$ can be fully recapitulated by microinjection of a single Mcrs 1 siRNA, resulting in severely reduced/no epiblast cells remaining in the absence of MCRS1 function.

\section{Discussion}

Lineage specification and subsequent cell differentiation is a complex and highly regulated process that occurs during the development of all multicellular eukaryotic organisms. Although the localization of specific transcription factors within ICM/TE and EPI/PE/TE lineages have been well studied during mammalian development, the upstream regulation of these critical factors is not fully understood (Paul \& Knott 2014, Marcho et al. 2015, Cui \& Mager 2018). Among the multiple signaling networks involved in early mammalian lineage specification, Hippo signaling has been shown to be involved in both TE (Strumpf et al. 2005, Yagi et al. 2007) and ICM (Wicklow et al. 2014) specification, while roles for Notch (Rayon et al. 2014) and ROCK signaling in TE fate acquisition have also been well supported (Kono et al. 2014, Negron-Perez et al. 2018). In addition, multiple factors are known to contribute to cell lineage segregation. For example, during mouse preimplantation, PRDM14 interacts with the methyltransferase CARM1 to promote methylation of arginine 26 of histone H3 (Burton et al. 2013). This interaction leads to an upregulation of NANOG and SOX2, biasing the progeny toward the ICM fate (TorresPadilla et al. 2007). Explaining these observations, two newer studies have found that H3R26me potentiates 


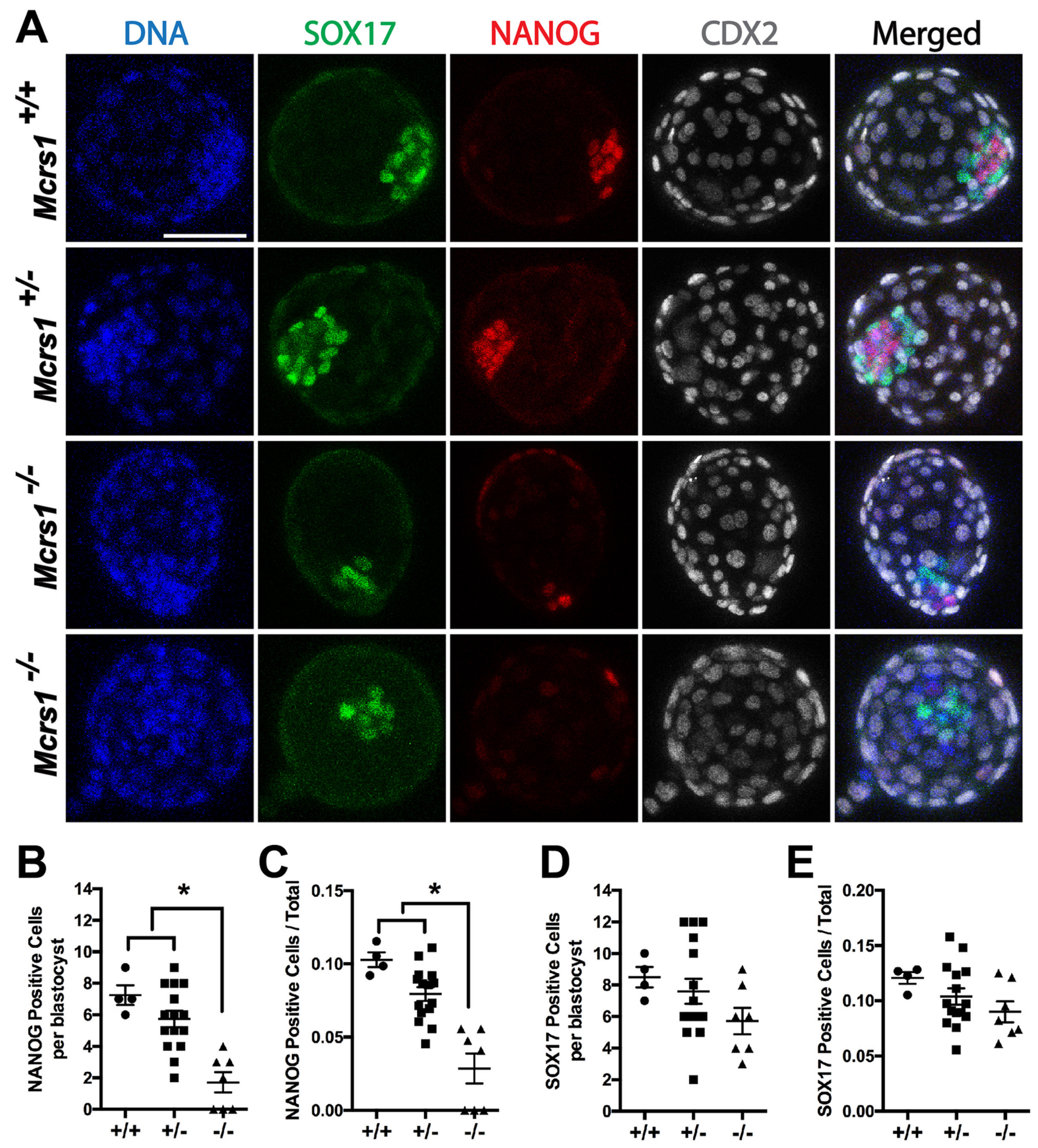

Figure 4 (A) IF of SOX17 (PE marker), NANOG (EPI marker), and CDX2 (TE marker) in blastocysts of different genotypes. (B and C) Both the number of EPI cells (NANOG positive) per blastocyst and percentage of EPI cells were significantly decreased in Mut embryos when compared with WT and Het embryos. (D and E) No significant difference in the number of PE cells (SOX17 positive) per blastocyst or percentage of PE cells was detected between genotypes. Scale bar, $50 \mu \mathrm{m} .{ }^{*} P<0.05$.

long-lived SOX2-DNA binding, ensuring access of SOX2 to its pluripotency-related targets while suppressing TE-specific genes, ultimately biasing blastomeres toward ICM (Goolam et al. 2016, White et al. 2016).
Recent single-cell expression profiling of human blastocyst-derived cells predicted that MCRS1 plays a role in EPI formation; however, its function during mammalian embryogenesis has not been studied in 

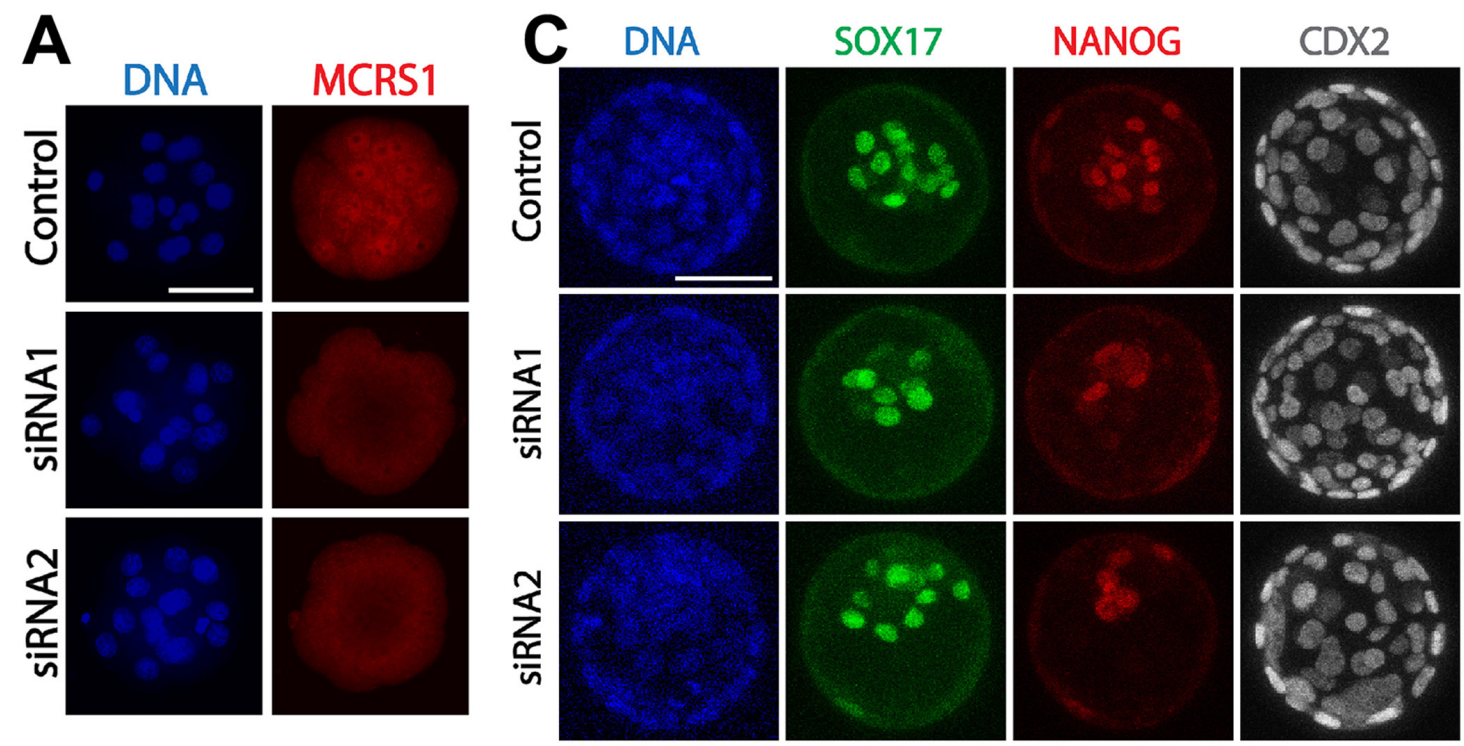

Merged

B

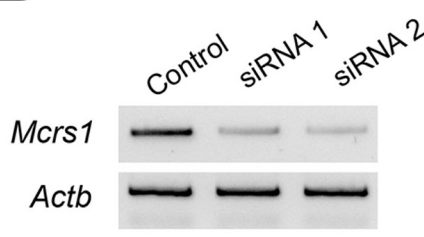

D

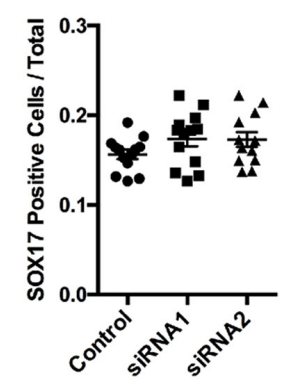

SiRNA1
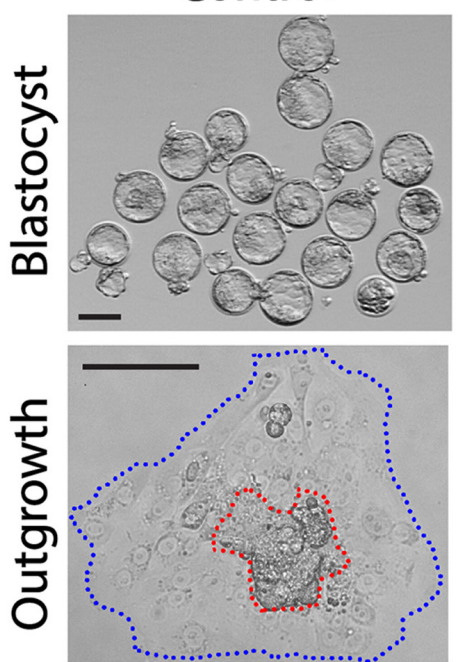

Control
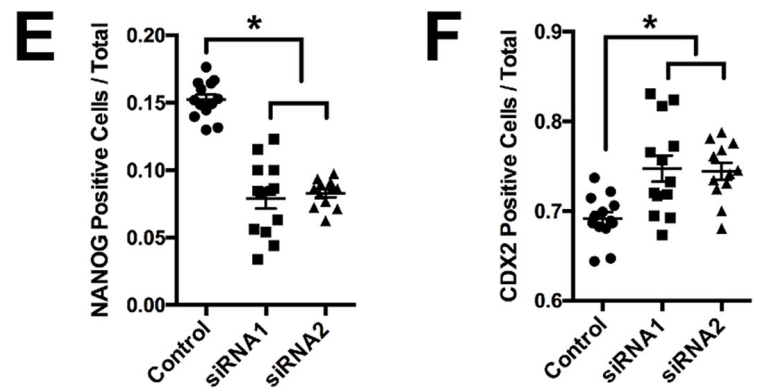

$\mathbf{G}$
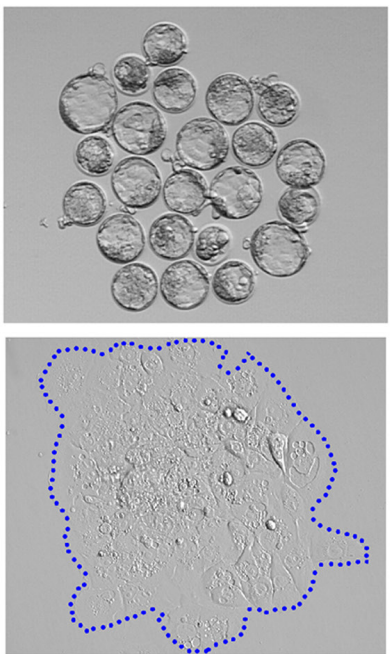

SiRNA2
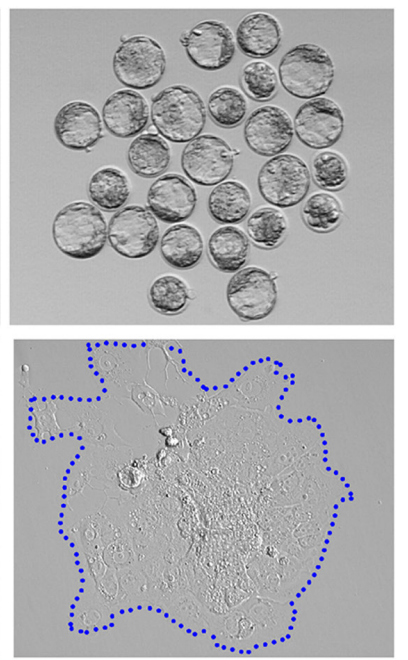
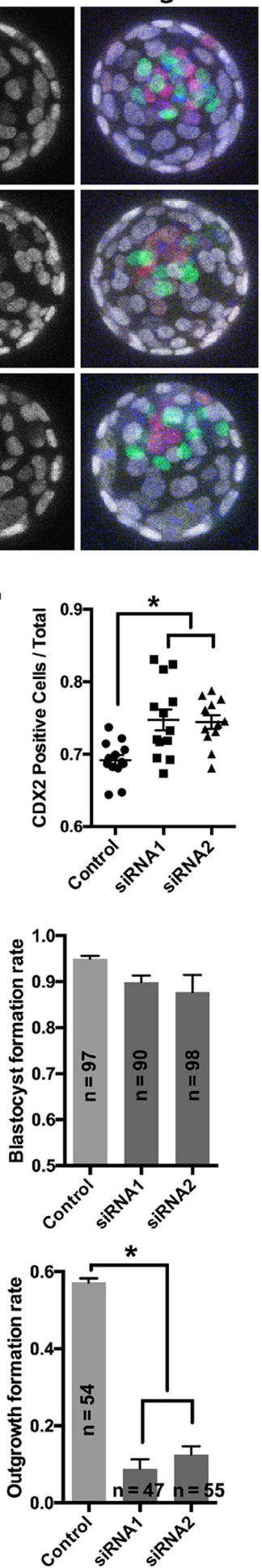

Figure 5 ( $A$ and B) KD efficiency was confirmed at the early morula stage (2 days after microinjection) by both MCRS1 protein IF and RT-PCR. Scale bar, $50 \mu \mathrm{m}$. (C, D, E and F) Lineage assessment in KD blastocysts showed that, compared with the Control group, Mcrs 1 KD did not alter the ratio of blastocyst cells with SOX17 (PE marker) or CDX2 (TE marker). However, as with KO of Mcrs1, the ratio of cells with NANOG (EPI marker) was decreased in each KD group. Scale bar, $50 \mu \mathrm{m}$. Blastocysts were harvested at 4 days post microinjection and then fixed for immunofluorescence. (G) KD of Mcrs 1 by either siRNA did not affect blastocyst formation or morphology, but led to a significant failure of ICM outgrowth. Red and blue dashed lines indicate ICM outgrowth and trophoblast cells, respectively. Control: scrambled siRNA. $n$, number of embryos; ${ }^{*} P<0.05$. Scale bars, $100 \mu \mathrm{m}$. 

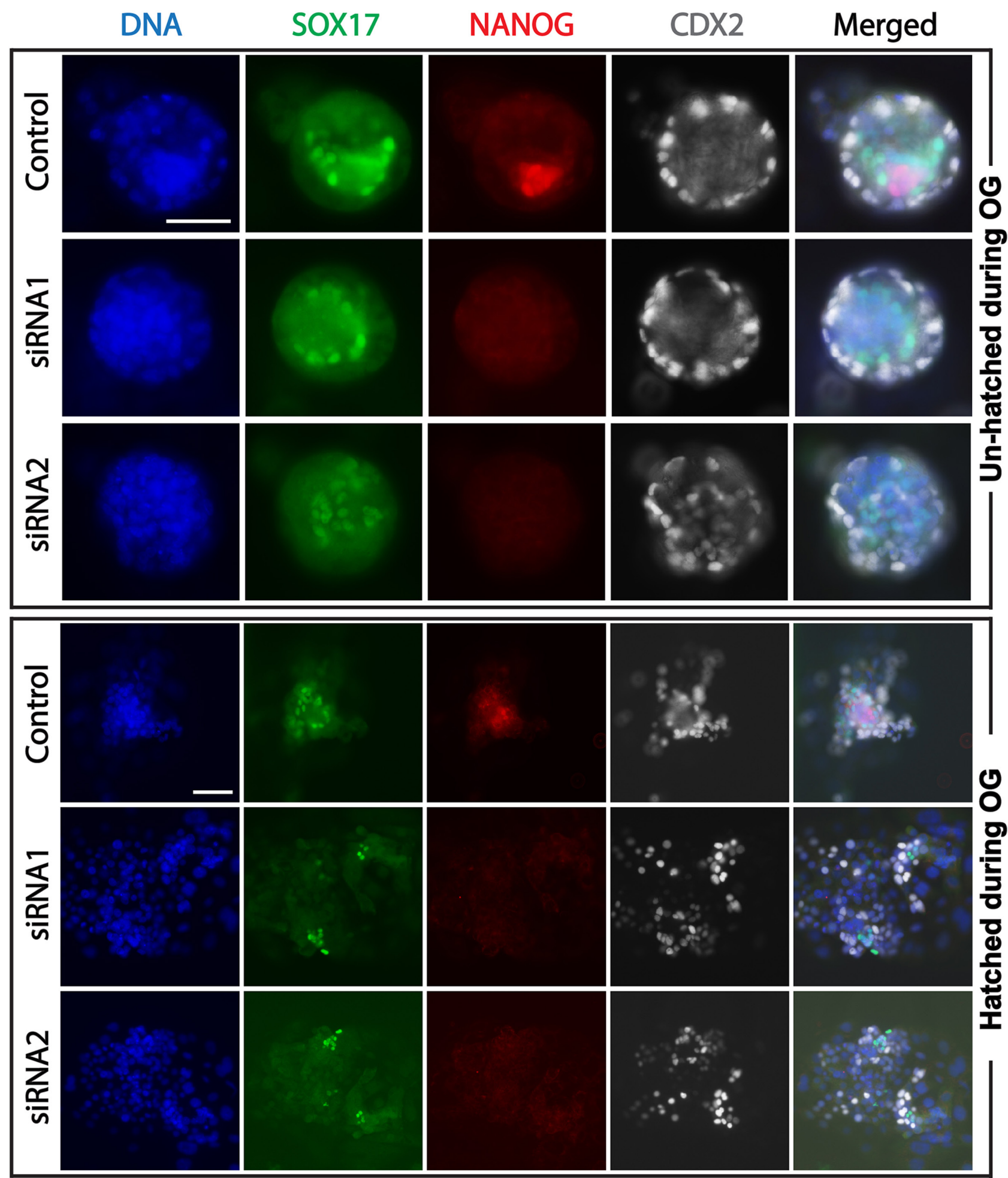

Figure 6 Lineage assessment of control and KD embryos that were subjected to an additional 72 h of outgrowth (OG) culture. No NANOGpositive cells could be detected in any KD embryo, regardless of failure to hatch. The control group consistently displayed strong NANOG signal. Control: scrambled siRNA. In this experiment blastocysts were examined at 7 days post microinjection. Scale bars, $50 \mu \mathrm{m}$. 
vivo (Durruthy-Durruthy et al. 2016). In the present study, we use $\mathrm{KO}$ and $\mathrm{KD}$ studies in vivo and in vitro to demonstrate the essential role of MCRS1 during mammalian embryogenesis. Our data show that Mcrs 1 mutant mouse embryos fail to implant and are unable to form ICM/epiblast colonies in outgrowth assays. Although previous studies using human cell lines have shown that MCRS1 silencing leads to apoptosis (Hsu et al. 2012), we do not observe increased cell death in mutant embryos (Fig. 3A), suggesting that MCRS1 regulates cell allocation rather than cell survival in vivo. Furthermore, because MCRS1 is a subunit of the NSL complex which can acetylate nucleosomal histone $\mathrm{H} 4$ on multiple lysine residues (Cai et al. 2010), we examined if loss of MCRS1 led to a reduction in $\mathrm{H} 4$ acetylation. IF analysis revealed that $\mathrm{KO}$ of Mcrs 1 causes no significant global difference in $\mathrm{H} 4$ acetylation ( $\mathrm{H} 4 \mathrm{~K} 5+\mathrm{K} 8+\mathrm{K} 12+\mathrm{K} 16)$ in the ICM of MCRS1 mutants (Fig. 3E). It is notable that the ENCODE database shows overlapping peaks of H4K8Ac and K4K91AC at promoters of OCT4 and NANOG but not SOX17 in hESC ChIP-seq experiments (https:// www.encodeproject.org). These findings are consistent with the hypothesis that MCRS1 does indeed facilitate $\mathrm{H} 4 \mathrm{Kac}$ deposition and gene activity in the ICM/EPI and that a more sensitive locus-specific assay may be required to detect consequences of MCRS1 loss in vivo. siRNA KD of MCRS1 may allow for production of large numbers of embryos with which to perform such ChIPseq experiments.

Our preliminary data show that knockout of Mcrs 1 did not alter cell lineage specification at E3.5 early blastocyst stage (Supplementary Fig. 4). We suspect this is due to maternally loaded MCRS1. It is also noteworthy that we used OCT4 as a definitive ICM marker (Fig. 3). However, it has been shown that loss of Oct4 does not affect formation of ICM (Nichols et al. 1998), therefore, it is possible that there could be OCT4-negative ICM cells. Thorough analysis of all observed blastocysts revealed that all cells are either OCT4 or CDX2 positive and we did not detect any OCT4 negative ICM cells (Fig. 3). These results also highlight an important difference with other epiblast-failure phenotypes (TCF7L1 (Hoffman et al. 2013) and FOXD3 (Hanna et al. 2002)), in that Mcrs $1 \mathrm{KO}$ embryos do specify ICM/TE normally but with far fewer ICM cells.

Our results clearly show a significant reduction of ICM cells, a reduction of NANOG-positive epiblast in E4.0 Muts and a complete loss of NANOG-positive cells in outgrowth assays in the absence of MCRS1 function. Importantly, TE and PE specification occur normally, highlighting the specific requirement of MCRS1 for epiblast specification or maintenance in murine blastocysts. It has been reported that a minimum of four NANOG-positive EPI cells are needed at the blastocyst stage for normal embryo development (Morris et al. 2012). Consistent with this report, our data show that all WTs (4/4) and majority of Hets (13/15) reached and surpassed this threshold, while only one of seven Mcrs 1 mutants obtained a minimal number of four NANOGpositive cells (1/7, Fig. 4B). It is noteworthy that MCRS1 protein shows ICM-enriched but not ICM-exclusive expression/localization pattern in mouse blastocysts, which is consistent with immunostaining of MCRS1 in human blastocysts (Durruthy-Durruthy et al. 2016). Therefore, we hypothesize that MCRS1 has multiple functions during embryo development. Previous studies have also confirmed that MCRS1 can be detected at different regions of the cell to perform different roles, such as in nucleolus to regulate rRNA transcription (Ren et al. 1998), in the nucleus to control gene transcription (Du et al. 2006, Andersen et al. 2010), on the centrosome to modulate mitosis and cell cycle transition (Hirohashi et al. 2006), as well as in the cytoplasm to regulate mRNA translation (Davidovic et al. 2006). While MCRS1 may not directly regulate global histone acetylation, we hypothesize that MCRS1 may directly regulate histone acetylation at transcription factors loci that are essential for EPI specification and maintenance. Documented MCRS1 function during induction of naive pluripotency in human ESCs (Durruthy-Durruthy et al. 2016) and its regulation of stem cell marker ITGA6 in cancer cells (Liu et al. 2014) support this hypothesis. Together, our study demonstrates that MCRS1 is essential for embryo survival by promoting the epiblast lineage during early murine embryogenesis in vivo and in vitro.

\section{Supplementary materials}

This is linked to the online version of the paper at https://doi.org/10.1530/REP-19-0334.

\section{Declaration of interest}

The authors declare that there is no conflict of interest that could be perceived as prejudicing the impartiality of the research reported.

\section{Funding}

This work was supported by R01HD083311 to J M and faculty start-up fund to W C.

\section{Author contribution statement}

W C designed the experiments, performed the majority of the experiments, analyzed the data and wrote the manuscript; A C conducted gastrulation embryo imaging and genotyping; $Y \mathrm{~W}$ participated in preimplantation embryo immunofluorescence and outgrowth assays; Y T contributed to mouse maintenance and genotyping; $\mathrm{Y} \mathrm{L}$ and $\mathrm{K} \mathrm{D} \mathrm{T}$ participated in critical discussion and edited the manuscript; J $M$ participated in experimental design, embryo dissection, data analysis and edited the manuscript. All authors reviewed the manuscript. 


\section{Acknowledgements}

The authors thank the Knockout Mouse Phenotyping Program (KOMP2) and The Jackson Laboratory for providing Mcrs1knockout allele. They also thank Morgane Golan for assistance in genotyping. The confocal microscopy data were gathered in the Light Microscopy Facility and Nikon Center of Excellence at the Institute for Applied Life Sciences, UMass Amherst with support from the Massachusetts Life Sciences Center.

\section{References}

An Q, Peng W, Cheng Y, Lu Z, Zhou C, Zhang Y \& Su J 2019 Melatonin supplementation during in vitro maturation of oocyte enhances subsequent development of bovine cloned embryos. Journal of Cellular Physiology 234 17370-17381. (https://doi.org/10.1002/jcp.28357)

Andersen DS, Raja SJ, Colombani J, Shaw RL, Langton PF, Akhtar A \& Tapon N 2010 Drosophila MCRS2 associates with RNA polymerase II complexes to regulate transcription. Molecular and Cellular Biology $\mathbf{3 0}$ 4744-4755. (https://doi.org/10.1128/MCB.01586-09)

Arny M, Nachtigall L \& Quagliarello J 1987 The effect of preimplantation culture conditions on murine embryo implantation and fetal development. Fertility and Sterility 48 861-865. (https://doi.org/10.1016/ s0015-0282(16)59545-4)

Benavides M, Chow-Tsang LF, Zhang J \& Zhong H 2013 The novel interaction between microspherule protein Msp58 and ubiquitin E3 ligase EDD regulates cell cycle progression. Biochimica and Biophysica Acta 1833 21-32. (https://doi.org/10.1016/j.bbamcr.2012.10.007)

Burton A, Muller J, Tu S, Padilla-Longoria P, Guccione E \& TorresPadilla ME 2013 Single-cell profiling of epigenetic modifiers identifies PRDM14 as an inducer of cell fate in the mammalian embryo Cell Reports. 5 687-701. (https://doi.org/10.1016/j.celrep.2013.09.044)

Cai Y, Jin J, Swanson SK, Cole MD, Choi SH, Florens L, Washburn MP, Conaway JW \& Conaway RC 2010 Subunit composition and substrate specificity of a MOF-containing histone acetyltransferase distinct from the male-specific lethal (MSL) complex. Journal of Biological Chemistry 285 4268-4272. (https://doi.org/10.1074/jbc.C109.087981)

Cao Z, Carey TS, Ganguly A, Wilson CA, Paul S \& Knott JG 2015 Transcription factor AP-2gamma induces early $\mathrm{Cdx} 2$ expression and represses HIPPO signaling to specify the trophectoderm lineage. Development 142 1606-1615. (https://doi.org/10.1242/dev.120238)

Cui W \& Mager J 2018 Transcriptional regulation and genes involved in first lineage specification during preimplantation development. Advances in Anatomy, Embryology, and Cell Biology 229 31-46. (https:// doi.org/10.1007/978-3-319-63187-5_4)

Cui W, Dai X, Marcho C, Han Z, Zhang K, Tremblay KD \& Mager J 2016 Towards functional annotation of the preimplantation transcriptome: an RNAi screen in mammalian embryos. Scientific Reports 637396. (https://doi.org/10.1038/srep37396)

Cui W, Marcho C, Wang Y, Degani R, Golan M, Tremblay KD, Rivera-Perez J \& Mager J 2018 Med20 is essential for early embryogenesis and regulates Nanog expression. Reproduction 157 215-222. (https:// doi.org/10.1530/REP-18-0508)

Davidovic L, Bechara E, Gravel M, Jaglin XH, Tremblay S, Sik A, Bardoni B \& Khandjian EW 2006 The nuclear microspherule protein 58 is a novel RNA-binding protein that interacts with fragile $\mathrm{X}$ mental retardation protein in polyribosomal mRNPs from neurons. Human Molecular Genetics 15 1525-1538. (https://doi.org/10.1093/hmg/ddl074)

Du X, Wang Q, Hirohashi Y \& Greene MI 2006 DIPA, which can localize to the centrosome, associates with p78/MCRS1/MSP58 and acts as a repressor of gene transcription. Experimental and Molecular Pathology 81 184-190. (https://doi.org/10.1016/j.yexmp.2006.07.008)

Durruthy-Durruthy J, Wossidlo M, Pai S, Takahashi Y, Kang G, Omberg L, Chen B, Nakauchi H, Reijo Pera R \& Sebastiano V 2016 Spatiotemporal reconstruction of the human blastocyst by single-cell gene-expression analysis informs induction of naive pluripotency. Developmental Cell $\mathbf{3 8}$ 100-115. (https://doi.org/10.1016/j.devcel.2016.06.014)

Fawal MA, Brandt M \& Djouder N 2015 MCRS1 binds and couples Rheb to amino acid-dependent mTORC1 activation. Developmental Cell 33 67-81. (https://doi.org/10.1016/j.devcel.2015.02.010)
Goolam M, Scialdone A, Graham SJL, Macaulay IC, Jedrusik A, Hupalowska A, Voet T, Marioni JC \& Zernicka-Goetz M 2016 Heterogeneity in Oct4 and Sox2 targets biases cell fate in 4-cell mouse embryos. Cell 165 61-74. (https://doi.org/10.1016/j. cell.2016.01.047)

Hanna LA, Foreman RK, Tarasenko IA, Kessler DS \& Labosky PA 2002 Requirement for Foxd3 in maintaining pluripotent cells of the early mouse embryo. Genes and Development 16 2650-2661. (https://doi. org/10.1101/gad.1020502)

Hirohashi Y, Wang Q, Liu Q, Du X, Zhang H, Sato N \& Greene MI 2006 p78/MCRS1 forms a complex with centrosomal protein Nde1 and is essential for cell viability. Oncogene 25 4937-4946. (https://doi. org/10.1038/sj.onc.1209500)

Hoffman JA, Wu CI \& Merrill BJ 2013 Tcf7l1 prepares epiblast cells in the gastrulating mouse embryo for lineage specification. Development $\mathbf{1 4 0}$ 1665-1675. (https://doi.org/10.1242/dev.087387)

Houliston E \& Maro B 1989 Posttranslational modification of distinct microtubule subpopulations during cell polarization and differentiation in the mouse preimplantation embryo. Journal of Cell Biology $\mathbf{1 0 8}$ 543-551. (https://doi.org/10.1083/jcb.108.2.543)

Hsu CC, Lee YC, Yeh SH, Chen CH, Wu CC, Wang TY, Chen YN, Hung LY, Liu YW, Chen HK et al. 2012 58-kDa microspherule protein (MSP58) is novel Brahma-related gene 1 (BRG1)-associated protein that modulates p53/p21 senescence pathway. Journal of Biological Chemistry 287 22533-22548. (https://doi.org/10.1074/jbc.M111.335331)

Jin J, Cai Y, Yao T, Gottschalk AJ, Florens L, Swanson SK, Gutierrez JL, Coleman MK, Workman JL, Mushegian A et al. 2005 A mammalian chromatin remodeling complex with similarities to the yeast INO80 complex. Journal of Biological Chemistry 280 41207-41212. (https:// doi.org/10.1074/jbc.M509128200)

Kono K, Tamashiro DA \& Alarcon VB 2014 Inhibition of Rho-ROCK signaling enhances ICM and suppresses TE characteristics through activation of Hippo signaling in the mouse blastocyst. Developmental Biology 394 142-155. (https://doi.org/10.1016/j.ydbio.2014.06.023)

Latham KE, Solter D \& Schultz RM 1991 Activation of a two-cell stagespecific gene following transfer of heterologous nuclei into enucleated mouse embryos. Molecular Reproduction and Development 30 182-186. (https://doi.org/10.1002/mrd.1080300303)

Liu MX, Zhou KC \& Cao Y 2014 MCRS1 overexpression, which is specifically inhibited by miR-129*, promotes the epithelial-mesenchymal transition and metastasis in non-small cell lung cancer. Molecular Cancer 13245. (https://doi.org/10.1186/1476-4598-13-245)

Liu Y, Wu F, Zhang L, Wu X, Li D, Xin J, Xie J, Kong F, Wang W, Wu Q et al. 2018 Transcriptional defects and reprogramming barriers in somatic cell nuclear reprogramming as revealed by single-embryo RNA sequencing. BMC Genomics 19 734. (https://doi.org/10.1186/ s12864-018-5091-1)

Marcho C, Cui W \& Mager J 2015 Epigenetic dynamics during preimplantation development. Reproduction 150 R109-R120. (https:// doi.org/10.1530/REP-15-0180)

Morris SA, Guo Y \& Zernicka-Goetz M 2012 Developmental plasticity is bound by pluripotency and the Fgf and Wnt signaling pathways. Cell Reports 2 756-765. (https://doi.org/10.1016/j.celrep.2012.08.029)

Negron-Perez VM, Rodrigues LT, Mingoti GZ \& Hansen PJ 2018 Role of ROCK signaling in formation of the trophectoderm of the bovine preimplantation embryo. Molecular Reproduction and Development 85 374-375. (https://doi.org/10.1002/mrd.22976)

Nichols J, Zevnik B, Anastassiadis K, Niwa H, Klewe-Nebenius D, Chambers I, Scholer H \& Smith A 1998 Formation of pluripotent stem cells in the mammalian embryo depends on the POU transcription factor Oct4. Cell 95 379-391. (https://doi.org/10.1016/s00928674(00)81769-9)

Niwa H, Toyooka Y, Shimosato D, Strumpf D, Takahashi K, Yagi R \& Rossant J 2005 Interaction between Oct3/4 and $\mathrm{Cdx} 2$ determines trophectoderm differentiation. Cell $\mathbf{1 2 3}$ 917-929. (https://doi. org/10.1016/j.cell.2005.08.040)

Paul S \& Knott JG 2014 Epigenetic control of cell fate in mouse blastocysts: the role of covalent histone modifications and chromatin remodeling. Molecular Reproduction and Development 81 171-182. (https://doi. org/10.1002/mrd.22219)

Rayon T, Menchero S, Nieto A, Xenopoulos P, Crespo M, Cockburn K, Cañon S, Sasaki H, Hadjantonakis AK, de la Pompa JL et al. 2014 Notch 
and hippo converge on $\mathrm{Cdx} 2$ to specify the trophectoderm lineage in the mouse blastocyst. Developmental Cell 30 410-422. (https://doi. org/10.1016/j.devcel.2014.06.019)

Ren Y, Busch RK, Perlaky L \& Busch H 1998 The 58-kDa microspherule protein (MSP58), a nucleolar protein, interacts with nucleolar protein p120. European Journal of Biochemistry 253 734-742. (https://doi. org/10.1046/j.1432-1327.1998.2530734.x)

Schneider CA, Rasband WS \& Eliceiri KW 2012 NIH Image to Image): 25 years of image analysis. Nature Methods 9 671-675. (https://doi. org/10.1038/nmeth.2089)

Strumpf D, Mao CA, Yamanaka Y, Ralston A, Chawengsaksophak K, Beck F \& Rossant J 2005 Cdx2 is required for correct cell fate specification and differentiation of trophectoderm in the mouse blastocyst. Development 132 2093-2102. (https://doi.org/10.1242/dev.01801)

Sutherland AE \& Calarco-Gillam PG 1983 Analysis of compaction in the preimplantation mouse embryo. Developmental Biology 100 328-338. (https://doi.org/10.1016/0012-1606(83)90227-0)

Tompers DM, Foreman RK, Wang Q, Kumanova M \& Labosky PA 2005 Foxd3 is required in the trophoblast progenitor cell lineage of the mouse embryo. Developmental Biology 285 126-137. (https://doi. org/10.1016/j.ydbio.2005.06.008)

Torres-Padilla ME, Parfitt DE, Kouzarides T \& Zernicka-Goetz M 2007 Histone arginine methylation regulates pluripotency in the early mouse embryo. Nature 445 214-218. (https://doi.org/10.1038/nature05458)
Tremblay KD, Hoodless PA, Bikoff EK \& Robertson EJ 2000 Formation of the definitive endoderm in mouse is a Smad2-dependent process. Development 127 3079-3090.

White MD, Angiolini JF, Alvarez YD, Kaur G, Zhao ZW, Mocskos E, Bruno L, Bissiere S, Levi V \& Plachta N 2016 Long-lived binding of Sox2 to DNA predicts cell fate in the four-cell mouse embryo. Cell 165 75-87. (https://doi.org/10.1016/j.cell.2016.02.032)

Wicklow E, Blij S, Frum T, Hirate Y, Lang RA, Sasaki H \& Ralston A 2014 HIPPO pathway members restrict SOX2 to the inner cell mass where it promotes ICM fates in the mouse blastocyst. PLoS Genetics 10 e1004618. (https://doi.org/10.1371/journal.pgen.1004618)

Yagi R, Kohn MJ, Karavanova I, Kaneko KJ, Vullhorst D, DePamphilis ML \& Buonanno A 2007 Transcription factor TEAD4 specifies the trophectoderm lineage at the beginning of mammalian development. Development $\mathbf{1 3 4} \quad 3827-3836 . \quad$ (https://doi. org/10.1242/dev.010223)

Received 24 July 2019

First decision 3 September 2019

Revised manuscript received 3 October 2019

Accepted 29 October 2019 